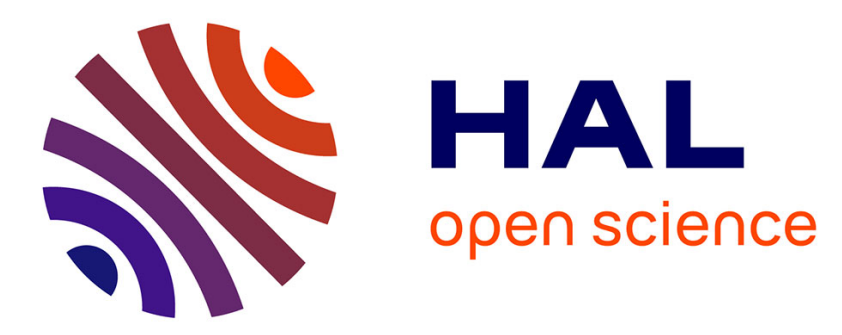

\title{
Modeling of the processes of ionization and excitation of nitrogen molecules by short and intense laser pulses
}

Vladimir T Tikhonchuk, Yi Liu, Rostyslav Danylo, Aurélien Houard, André Mysyrowicz

\section{- To cite this version:}

Vladimir T Tikhonchuk, Yi Liu, Rostyslav Danylo, Aurélien Houard, André Mysyrowicz. Modeling of the processes of ionization and excitation of nitrogen molecules by short and intense laser pulses. Physical Review A, 2021, 104 (6), pp.063116. 10.1103/PhysRevA.104.063116 . hal-03510632

\section{HAL Id: hal-03510632 \\ https://hal-ensta-paris.archives-ouvertes.fr/hal-03510632}

Submitted on 4 Jan 2022

HAL is a multi-disciplinary open access archive for the deposit and dissemination of scientific research documents, whether they are published or not. The documents may come from teaching and research institutions in France or abroad, or from public or private research centers.
L'archive ouverte pluridisciplinaire HAL, est destinée au dépôt et à la diffusion de documents scientifiques de niveau recherche, publiés ou non, émanant des établissements d'enseignement et de recherche français ou étrangers, des laboratoires publics ou privés. 


\title{
Modeling of the processes of ionization and excitation of nitrogen molecules by short and intense laser pulses
}

\author{
Vladimir T. Tikhonchuk, ${ }^{1,2}$ Yi Liu, ${ }^{3,4}$ Rostyslav Danylo, ${ }^{5,3}$ Aurélien Houard ${ }^{5}$ and André Mysyrowicz ${ }^{5}$ \\ ${ }^{1}$ University of Bordeaux-CNRS-CEA, CELIA, UMR 510\%, 33405 Talence, France* \\ ${ }^{2}$ ELI-Beamlines Center, Institute of Physics, Czech Academy of Sciences, 25241 Dolni Břežany, Czech Republic \\ ${ }^{3}$ Shanghai Key Lab of Modern Optical System, University of Shanghai for Science and Technology, 200093 Shanghai, China \\ ${ }^{4}$ CAS Center for Excellence in Ultra-intense Laser Science, Shanghai, 201800, China \\ ${ }^{5}$ Laboratoire d'Optique Appliquée, ENSTA Paris, CNRS, Ecole Polytechnique, \\ Institut Polytechnique de Paris, 91762 Palaiseau, France
}

(Dated: November 24, 2021)

\begin{abstract}
We present a model describing ionization and excitation of nitrogen molecules by a strong and short laser pulse. In difference to previous publications, both processes are considered within the same formalism of density matrix. We account for the dependence of the dipole moment on the vibrational quantum number and for a large number of excited levels. Populations of the excited levels depend significantly on the laser intensity, wavelength, polarization and pulse duration.
\end{abstract}

\section{INTRODUCTION}

Cavity-free lasing of nitrogen singly-ionized molecules has been reported for the first time 10 years ago $[1,2]$ and attracted the attention of many research groups [39]. The effect consists in lasing in the forward direction at the wavelengths 391 or $428 \mathrm{~nm}$, corresponding to transitions from the excited state $\mathrm{B}^{2} \Sigma_{u}^{+}$to the ground states $\mathrm{X}^{2} \Sigma_{g}^{+}(0,1)$ of $\mathrm{N}_{2}^{+}$molecule with vibrational levels 0 or 1 , respectively. There is still an ongoing controversy on how this lasing is produced as, owing to a very short duration of the main laser pulse, typically less than $50 \mathrm{fs}$, it is not known whether population inversion between $\mathrm{B}$ and X states can be created.

This controversy in the explanation of the lasing effect is due to the fact that the nitrogen molecule is a complex quantum object and only a limited number of excited levels is often considered. The challenge consists in evaluating the probability of excitation of different electronic and vibrational levels after the end of the laser pulse. Only knowing the populations at all relevant levels it would be possible to define the origin of the lasing process.

The probability of the direct laser ionization into excited state B is very low compared to ionization into ground state X0 because of the large difference of energies and the low probability of electron recollision $[10,11]$. However, there are three other processes, which may contribute to the observed lasing effect. The first process is the excitation to a third level A. There is an intermediate state $\mathrm{A}^{2} \Pi_{u}$ between the $\mathrm{X}$ and $\mathrm{B}$ states that can be resonantly excited by the main pulse because the transition energy between X0 and A2 levels, $\hbar\left(\omega_{a 2}-\omega_{x 0}\right)=1.59 \mathrm{eV}$ is very close to the pump photon energy, $\hbar \omega_{0}=1.55 \mathrm{eV}$. Thus, a transition of a fraction of ionized molecules to the A state may create a population inversion between

\footnotetext{
* tikhonchuk@u-bordeaux.fr
}

$\mathrm{B}$ and $\mathrm{X}$ states and explain the lasing effect $[5,6]$. However, this scheme assumes a population inversion between the $\mathrm{A}$ and $\mathrm{X}$ states, which is not evidenced experimentally and is not found in the numerical modeling [12]. A second process is the polarization coupling between $\mathrm{B}$ and $\mathrm{A}$ states [13]. This process, known also as the V-scheme [14-16], can explain the lasing effect without population inversion $[12,13,17-20]$. The third process is the population inversion between $\mathrm{B}$ and $\mathrm{X}$ states because of coherent reconstruction of rotational wave packet of $\mathrm{N}_{2}^{+}$ions [9, 21]. Indeed, ionization of nitrogen molecules is accompanied by molecular rotations, which correspond to a coherent excitation of many rotational levels. The periodic alignment of the molecular ions results in a transient inversion between certain rotational $\mathrm{B}$ and $\mathrm{X}$ levels that may explain the lasing effect $[22,23]$. However, such inversion exists only for short time periods of molecular alignment separated by a half of period of molecular rotations, which is equal to approximately $4 \mathrm{ps}$. The predicted gain is too small and incompatible with the observations.

In our previous paper [12], we have considered a model of the nitrogen molecular ion $\mathrm{N}_{2}^{+}$including the ground electronic state $\mathrm{X}^{2} \Sigma_{g}^{+}$with two vibrational levels $v=0,1$ and two excited electronic levels: first excited state $\mathrm{A}^{2} \Pi_{u}$ with two vibrational levels $v=2,3$ and second excited state $\mathrm{B}^{2} \Sigma_{u}^{+}$with one vibrational level $v=0$. The model was simplified by assuming a limited number of allowed transitions, namely, four transitions were considered, A2$\mathrm{X} 0, \mathrm{~A} 3-\mathrm{X} 1, \mathrm{~B} 0-\mathrm{X} 0$ and $\mathrm{B} 0-\mathrm{X} 1$, and two $\mathrm{A}-\mathrm{X}$ and two $\mathrm{B}-\mathrm{X}$ transitions were characterized by the same dipole moments, $\mu_{a x}=0.25 e a_{\mathrm{B}}$ and $\mu_{b x}=0.75 e a_{\mathrm{B}}[24,25]$, where $e$ is the unitary charge and $a_{\mathrm{B}}$ is the Bohr radius. This choice of transitions was justified by the resonance conditions as the frequencies of $\mathrm{A} 2-\mathrm{X} 0$ and $\mathrm{A} 3-\mathrm{X} 1$ transitions are close to the laser frequency.

Although this model goes beyond the traditional three level scheme [5, 6], it is not known how good this simplified five-level model corresponds to the reality as the nitrogen ion has much more vibrational levels and many 


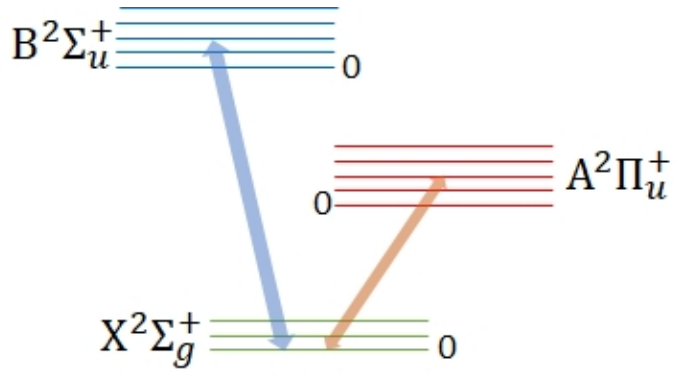

FIG. 1. Scheme of electronic and vibrational levels in the molecule $\mathrm{N}_{2}^{+}$. Number of lines indicates the number of vibrational levels considered, rotational splitting is neglected. Arrows show the allowed transitions.

more cross-couplings are allowed. Recent study by Zhang et al. [26] shows the possibility of efficient excitation of higher vibrational B-levels. In the present work we extend the ionization-excitation model by revising the ionization model of nitrogen molecule, including more vibrational levels at $\mathrm{X}$ and $\mathrm{A}$ states and accounting for all allowed transitions between the considered levels shown in figure 1. We consider the dependence of dipole moments on the vibrational quantum number by using experimental values for the Einstein coefficients for transitions and Franck-Condon coefficients for ionization [24, 25, 27, 28].

A comparison of populations of involved states for the realistic laser pulse parameters shows that it is necessary to account for $3-5$ vibrational levels in each electronic state in order to have convergent results. The calculated populations are significantly different from the simplified three- and five-level models and from the calculation of populations by using the equations for the probability amplitudes $[5,7]$.

\section{ELEMENTS OF THE THEORETICAL MODEL}

Our model is based on the Bloch equations for the density matrix of a nitrogen molecule in a prescribed strong, time-dependent laser field. In difference from equations for the complex probability amplitudes, which describe only transitions between discrete levels, equations for the density matrix may include also the terms describing the molecule ionization into the ground and excited ionic states. Thus, the use of equations for the density matrix allows one to account for both ionization and excitation processes self-consistently, which cannot be done with the equations for the probability amplitudes.

Some authors $[5,7,29,30]$ have proposed to associate ionization with the maximum of the laser pulse and then to solve the amplitude equations for the second half of the pulse for the evaluation of population at the excited levels. However, our analysis shows that such a scheme is inconsistent with the density matrix approach showing quantitatively different results for the ionization level and populations at excited states.

The dynamic equation for the density matrix $\mathbf{d}$ has a standard form $[12,26,31]$ :

$$
\dot{\mathbf{d}}=-\frac{i}{\hbar}[\mathbf{H}, \mathbf{d}]+\left(1-n_{i}\right) \mathbf{w}_{\mathrm{fi}},
$$

where the Poisson bracket in the first term on the right hand side describes transitions between the ground and excited states of the ion, and the second term describes ionization of the nitrogen molecule to the ground and excited states. The density matrix is normalized to the density of neutral molecules. Then, trace of $\mathbf{d}$ gives the total probability of ionization $n_{i}=\operatorname{Tr} \mathbf{d}$. According to equation (1), it satisfies equation

$$
\dot{n}_{i}=\left(1-n_{i}\right) \operatorname{Tr} \mathbf{w}_{\mathrm{fi}} .
$$

Ionization matrix $\mathbf{w}_{\mathrm{fi}}$ is diagonal and represents the probability of ionization to the correspondent levels.

Hamiltonian, $\mathbf{H}=\mathbf{H}^{0}-\boldsymbol{\mu} \cdot \mathbf{E}(t)$ is assumed to be Hermitian with the diagonal term $\mathbf{H}^{0}=\hbar \boldsymbol{\omega}$ representing the energies of the ground and excited states and the off-diagonal term $\boldsymbol{\mu} \cdot \mathbf{E}=\hbar \boldsymbol{\Omega}$ representing the dipole interaction between levels with $\Omega$ being the Rabi frequency. Explicit expressions for the ionization and dipole matrices for the 13 considered levels and 29 transitions are presented in the next sections.

We consider excited electronic states of the nitrogen molecular ion $\mathrm{A}^{2} \Pi_{u}$ and $\mathrm{B}^{2} \Sigma_{u}^{+}$corresponding to the value of the dominant orbital quantum number $l=1$ and magnetic quantum number (projection of the orbital momentum on the molecule axis) $m=0$ (B state) and $m= \pm 1$ (A state). Each of these states, as well as the ground state $\mathrm{X}^{2} \Sigma_{g}^{+}$, includes many vibrational levels. We consider three vibrational levels in the ground state $\mathrm{X}, v=0-2$, five levels in the A state, $v=0-4$ and five levels $v=0-4$ of the B state, which correspond to 29 transitions in the optical and near infrared range. The wavelengths of the considered transitions along with the corresponding Einstein coefficients are given in table II. In particular, the wavelengths of transitions $\mathrm{A} 2-\mathrm{X} 0$ and $\mathrm{A} 3-\mathrm{X} 1$ are very close to the laser wavelength of $800 \mathrm{~nm}$ used in many experiments and the wavelengths of transitions B0-X0 and $\mathrm{B} 0-\mathrm{X} 1$ are close to its second harmonic. Compared to Zhang et al. [26], we omitted higher order vibrational levels $v=3,4$ in X state as their populations are expected to be quite small, less than $0.1 \%$.

Expressions for the dipole interaction depend on the laser pulse polarization. In the case of linear polarization, it depends on the angle $\Theta$ between the directions of electric field and molecule axis. Considering the laser field directed along the $z$-axis in the form

$$
E_{z}(t)=E_{0} \cos \left(\omega_{0} t\right) \cos \left(\pi t / 2 t_{\text {las }}\right) H\left(t_{\text {las }}-|t|\right),
$$

where $E_{0}$ is the laser amplitude, $\omega_{0}$ is the laser frequency, $t_{\text {las }}$ is laser pulse duration at half maximum intensity and $H$ is the Heaviside function. The Rabi frequencies for the 
$\mathrm{B}-\mathrm{X}$ and $\mathrm{A}-\mathrm{X}$ transitions read:

$$
\begin{aligned}
& \hbar \Omega_{B X}=\mu_{B X} E_{z} \cos \Theta, \\
& \hbar \Omega_{A X}=\mu_{A X} E_{z} \sin \Theta,
\end{aligned}
$$

where $\hbar$ is the Planck constant. Considering a laser pulse duration in a tens of femtosecond scale, which is much smaller than the period of molecular rotation $(\sim 8 \mathrm{ps})$, we average the density matrix at the end of laser pulse over the molecular orientation with respect to the laser field direction, assuming there is no privileged orientation before the laser arrival. The spatial dependence of the laser field is not considered in this model as the laser pulse is assumed to be sufficiently strong and weakly modified while propagating in the gas. Consequently, all molecules are ionized and excited synchronously with the laser pulse propagation. Intensity in the calculations shown below corresponds to the maximum value, $I_{\text {las }}=\frac{1}{2} c \epsilon_{0} E_{0}^{2}$.

Calculation of the molecule ionization and excitation becomes more complicated if the laser polarization changes direction within pulse duration. Here, we consider a laser wave of elliptic polarization propagating in the $z$ direction and having electric field $\mathbf{E}$ in the $x, y$ plane composed of two linear polarizations, $\mathbf{E}(t)=$ $\mathbf{x} E_{x}(t)+\mathbf{y} E_{y}(t)$, defined by unit vectors $\mathbf{x}$ and $\mathbf{y}$, with the phases shifted by a quarter of the period:

$$
\begin{aligned}
& E_{x}(t)=E_{0} \cos \beta \cos \left(\omega_{0} t\right) \cos \left(\pi t / 2 t_{\text {las }}\right) H\left(t_{\text {las }}-|t|\right), \\
& E_{y}(t)=E_{0} \sin \beta \sin \left(\omega_{0} t\right) \cos \left(\pi t / 2 t_{\text {las }}\right) H\left(t_{\text {las }}-|t|\right) .
\end{aligned}
$$

A difference in amplitudes, $E_{0 x}$ and $E_{0 y}$, is controlled by the angle $\beta$ and ellipticity is defined as $\epsilon=\tan \beta$.

Molecule orientation is characterized by a unit vector $\mathbf{n}$, which in spherical coordinates is defined by the polar angle $\theta_{n}$ with respect to the laser propagation direction $z$ and the azimuthal angle $\psi_{n}$ in the laser polarization plane $x, y$. The absolute value of laser electric field and the angle between molecule orientation and laser field can be presented as

$$
\begin{aligned}
E_{\mathrm{abs}}(t) & =\left(E_{x}^{2}+E_{y}^{2}\right)^{1 / 2}, \\
\cos \Theta & =\sin \theta_{n}\left(\cos \psi_{n} \frac{E_{x}}{E_{\mathrm{abs}}}+\sin \psi_{n} \frac{E_{y}}{E_{\mathrm{abs}}}\right) .
\end{aligned}
$$

Rabi frequencies for $\mathrm{B}-\mathrm{X}$ transitions, with orbitals that have zero projection on the molecular axis are given by equation (4) with the field $E_{z}$ replaced by $E_{\text {abs }}$. More attention is required for the definition of Rabi frequencies for the A-X transitions, which correspond to a change of the projection of orbital momentum to the molecular axis $\Delta m= \pm 1$. Here, there are two probability amplitudes corresponding to left- and right-hand rotation. They are coupled to the corresponding field amplitudes $E^{ \pm}=2^{-1 / 2}\left(E_{x^{\prime}} \pm i E_{y^{\prime}}\right)$, which are defined in the coordinate system where molecular axis, $\mathbf{n}$, is oriented along the $z^{\prime}$-axis. We define the $y^{\prime}$ axis by a unit vector directed perpendicularly to the plane defined by the $z$-axis and the molecule axis: $\mathbf{y}^{\prime}=\mathbf{z} \times \mathbf{n} / \sin \theta_{n}$. Then, the third direction in this system, $\mathbf{x}^{\prime}$, is defined as a vector product, $\mathbf{x}^{\prime}=\mathbf{y}^{\prime} \times \mathbf{n}$. Consequently, the field components in the molecule reference system are defined by the following relations:

$$
E^{ \pm}=2^{-1 / 2} E_{x} \mathbf{x} \cdot\left(\mathbf{x}^{\prime} \pm i \mathbf{y}^{\prime}\right)+2^{-1 / 2} E_{y} \mathbf{y} \cdot\left(\mathbf{x}^{\prime} \pm i \mathbf{y}^{\prime}\right)
$$

In the particular case of time-independent laser polarization, we can assume that two vectors, laser polarization and molecule orientation, are lying in the $x, z$-plane, and then $\cos \Theta=\sin \theta_{n}$ and $E^{ \pm}=E \cos \theta_{n}$. In a more general case, the Rabi frequencies are also split into left- and right components:

$$
\hbar \Omega_{A X}^{ \pm}=\mu_{A X} E^{\mp} .
$$

This matrix is Hermitian: $\Omega_{A X}^{+}=\Omega_{A X}^{-\star}$.

\section{A. Dipole moments of electronic and vibrational transitions}

The transition wavelengths $\lambda_{a b}$ and the corresponding Einstein coefficients $A_{a b}$ are given in table II in Appendix A. The dipole moments are related to the Einstein coefficients as follows [32]:

$$
\mu_{a b}^{2}=\frac{3 \hbar \epsilon_{0}}{16 \pi^{2}} \lambda_{a b}^{3} A_{a b},
$$

where, $\epsilon_{0}$ is the vacuum dielectric permittivity.

Dipole moments for B-X transitions shown in table II are significantly larger than those for $\mathrm{A}-\mathrm{X}$ transitions. This leads to a larger population at B levels and smaller population at A levels, which favors the subsequent amplification in the V-scheme. Dipole moments for the considered states depend on the vibrational quantum number. This fact was not accounted for in previous publications $[5,12,29]$, which leads to quantitative differences in the populations of excited levels.

\section{B. Ionization probability}

Ionization of the nitrogen molecules is described by the model developed in references [33-35] and adapted for diatomic molecules by Tong et al. [36]. Ionization probability to level $a$ is characterized by the ionization potential $U_{a}$ and reads:

$$
\begin{aligned}
w_{a, \mathrm{fi}}= & \omega_{\mathrm{at}} \frac{q_{a}^{\mathrm{FCi}} B_{a}^{2}}{2^{|m|}|m| !} \kappa_{a}^{1-2 / \kappa_{a}} \\
& \times\left(\frac{2 \kappa_{a}^{3}}{F}\right)^{2 / \kappa_{a}-|m|-1} \exp \left(\frac{2 \kappa_{a}^{3}}{3 F}\right) .
\end{aligned}
$$

Here, $m$ is the projection of the orbital moment on the molecule axis, $\kappa_{a}=\left(2 U_{a} / \hbar \omega_{\mathrm{at}}\right)^{1 / 2}$ and $F=E_{\mathrm{abs}} / E_{\mathrm{at}}$ are the ionization energy and electric field expressed in atomic units, $\omega_{\mathrm{at}}=e^{2} / 4 \pi \epsilon_{0} a_{\mathrm{B}} \hbar$ and $E_{\mathrm{at}}=\hbar \omega_{\mathrm{at}} / e a_{\mathrm{B}}$, 

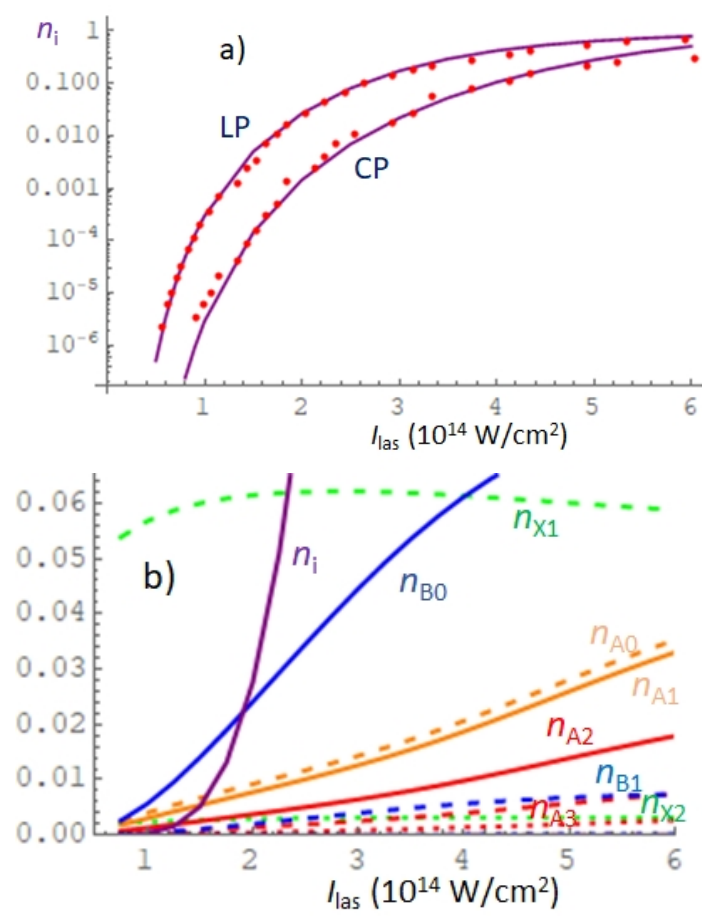

FIG. 2. a) Dependence of the total ionization probability $n_{i} / n_{0}$ on the laser intensity. Solid lines represent calculations with our model for linear (LP) and circular (CP) laser polarization. Points show the correspondent experimental results from reference [38]. b) Dependence of populations at excited states X1 (green, long dashed), X2 (green, dotted), A0 (orange, dashed), A1 (orange, solid), A2 (red, solid), A3 (red, dashed), A4 (red, dotted), B0 (blue, solid), B1 (blue, dashed), B2 (blue, dotted) normalized to the total ion density on the laser intensity for laser pulse duration $30 \mathrm{fs}$ and laser wavelength $800 \mathrm{~nm}$. Results obtained by solving equations for the density matrix (1) for 13 levels without polarization coupling between levels. Population at the level X0, which is on the order of $90 \%$, is not shown in the figure.

$q_{a}^{\mathrm{FCi}}$ is the Franck-Condon ionization factor [28] and coefficient $B_{a}$ is expressed as:

$$
B_{a}(m, \Theta)=\Sigma_{l, m^{\prime}} C_{l} D_{m^{\prime}, m}^{(l)} Q\left(l, m^{\prime}\right),
$$

where $Q(l, m)=(-1)^{m}[(2 l+1)(l+|m|) ! / 2(l-|m|) !]^{1 / 2}$ are the numerical coefficients, $D_{m^{\prime}, m}^{(l)}(\Theta)$ is the matrix of rotation [37] depending on the angle $\Theta$ between directions of electric field and molecule axis. The coefficients $C_{l}$ are provided by Zhang et al. in Supplementary material [26] and given in table III in Appendix A.

The angular dependence of the ionization probability is defined by the expression for the coefficients $B_{a}(m, \Theta)$ given by equation (14). Following table III, we assume that two orbitals, $l=0$ and $l=2$, contribute to the ground state X. For A and B states, it is sufficient to account for one orbital $l=1$. Consequently, by using data from table III, following expressions for coefficients $B$ can be found: $B_{X} \simeq 1+3 \cos ^{2} \Theta, B_{A} \simeq 5.3, B_{B} \simeq 5.2 \cos \Theta$.

\begin{tabular}{|c|c|c|c|c|c|c|c|c|c|c|c|}
\hline \multicolumn{2}{|c|}{ Level } & X0 & X1 & X2 & A0 & A1 & A2 & A3 & B0 & B1 & B2 \\
\hline \multirow{4}{*}{$\mathrm{N}$} & {$[26]$} & 0.560 & 0.049 & 0. & 0.066 & 0.081 & 0.057 & 0.030 & 0.005 & 0. & 0. \\
\cline { 2 - 13 } & $45^{\circ}$ & 0.773 & 0.057 & 0.003 & 0.064 & 0.057 & 0.029 & 0.012 & 0. & 0. & 0. \\
\cline { 2 - 13 } & Avr. & 0.836 & 0.062 & 0.003 & 0.017 & 0.015 & 0.008 & 0.003 & 0.050 & 0.004 & 0. \\
\hline \multirow{4}{*}{$\mathrm{P}$} & {$[26]$} & 0.064 & 0.058 & 0.019 & 0.141 & 0.174 & 0.186 & 0.059 & 0.084 & 0.023 & 0.009 \\
\cline { 2 - 12 } & $45^{\circ}$ & 0.043 & 0.087 & 0.016 & 0.056 & 0.061 & 0.075 & 0.042 & 0.095 & 0.191 & 0.091 \\
\cline { 2 - 11 } & Avr. & 0.097 & 0.164 & 0.027 & 0.036 & 0.053 & 0.130 & 0.046 & 0.067 & 0.161 & 0.111 \\
\hline
\end{tabular}

TABLE I. Comparison of the populations at the ground and excited levels calculated with the model presented in reference [26] and our model for the case without polarization coupling $(\mathrm{N})$ and with polarization coupling $(\mathrm{P})$. Laser intensity $3 \times 10^{14} \mathrm{~W} / \mathrm{cm}^{2}$, wavelength $800 \mathrm{~nm}$, pulse duration $30 \mathrm{fs}$. Lines " $45^{\circ}$ " and "Avr." show our calculations for $\Theta=45^{\circ}$ and angle averaged, respectively, for the same conditions.

This angular dependence of ionization is in qualitative agreement with the experimental results presented in reference [39].

This ionization model predicts, however, too high ionization probability compared to experiments [38]. This can be explained by the radial distribution of the laser pulse intensity in the experiment: the gas ionization averaged in the direction transverse to the propagation axis is smaller than at the axis. Agreement with the experiment can be achieved by reducing all ionization probabilities in equation (13) by a numerical factor of 10 . With this correction, as shown in figure $2 \mathrm{a}$, we find a good agreement of the angle-averaged ionization probability in the range of laser intensity $(0.5-6) \times 10^{14} \mathrm{~W} / \mathrm{cm}^{2}$ with the experimental data given by Guo et al. [38] for the laser pulse duration of $30 \mathrm{fs}$ full width at half maximum. Experimental data are normalized by a factor of $3.5 \times 10^{-8}$ and shifted on intensity by $1.4 \times 10^{13} \mathrm{~W} / \mathrm{cm}^{2}$. (This shift is within the precision of intensity definition in the experiment.) Agreement is also good for the circular laser polarization with the same scaling and shift factor as for the linear polarization. This close agreement gives us a confidence in considering the populations at excited states. Since the excitation probabilities do not depend on the absolute number of ionized molecules, we use this ionization suppression factor 0.1 in all calculations presented in below.

Figure $2 \mathrm{~b}$ shows the distribution of populations at the excited states as a function of laser intensity for the same pulse duration of $30 \mathrm{fs}$. Ionization to the ground state dominates with the fraction of ions decreasing from $93 \%$ at low intensity to about $78 \%$ at high intensity. Populations of all excited states are low and follow the inverse order to the ionization energy. This figure demonstrates that direct ionization into excited states cannot produce inversion of population and explain the lasing effect. Coupling between levels changes completely the situation.

The results of our ionization model can be compared with the population calculations performed by Zhang et al. [26] for the laser intensity $3 \times 10^{14} \mathrm{~W} / \mathrm{cm}^{2}$, wavelength $800 \mathrm{~nm}$, pulse duration of $30 \mathrm{fs}$ and for a particular angle 
of molecule orientation with respect to the laser polarization of $45^{\circ}$. The authors use a similar theoretical model based on solution of equation (1) for the density matrix. The comparison is shown in table I. In the case without polarization coupling, there is a qualitative agreement in the predicted populations for molecule orientation at $45^{\circ}$. Differences are more significant in the case where the populations are averaged over the molecule orientation and the polarization coupling is turned on. This is important for the levels X0, A2 and B0, which are of particular interest for amplified emission at the wavelength $391 \mathrm{~nm}$, corresponding to B0-X0 transition [13].

\section{IONIZATION-EXCITATION MODEL}

\section{A. Dependence on the number of excited levels}

Bloch equations (1) and (2) are solved numerically, ion state probabilities are evaluated at the end of the laser pulse, $t=2 t_{\text {las }}$, and averaged over the angle $\Theta$ assuming random orientation of molecules with respect to the laser electric filed. Figure 3a shows dependence of populations at the excited levels on the laser intensity for pulse duration $30 \mathrm{fs}$, wavelength of $800 \mathrm{~nm}$ and linear polarization obtained with the model including thirteen levels. Populations are strongly enhanced and reversed due to the polarization coupling compared to the case where only direct ionization is activated, figure $2 \mathrm{~b}$. Compared to the five-level model [12], ions are distributed more equally between several excited levels and populations oscillate as laser intensity increases.

Having in hand simulation results obtained with the model accounting for 13 levels and 29 transitions, we can now take a step back and evaluate the minimum number of levels and transitions needed for accurate calculation of populations. By suppressing transitions characterized by small values of dipole moment, we conclude that all transitions going to level X2 can be removed without significantly affecting populations at other levels. Similar observation applies to transitions going from levels A4, B3 and B4. In total 4 levels and 17 transitions can be suppressed in the model without affecting the populations at the remaining levels. Thus, the minimum configuration, includes two levels in X state $(v=0,1)$, four levels in A state $(v=0-3)$ and three levels in B state $(v=0-2)$. Dependence of populations on the laser intensity obtained with the reduced model is shown in figure 3b. A qualitative agreement with the full model shown in panel a) is evident, but there are quantitative differences that might be important for evaluation of inversion of population between some particular levels.

This analysis sheds also light on how the levels are populated. Figure 4 shows an example of temporal evolution of populations on several representative levels. Ionization populates essentially X0 level. Level X1 is populated through intermediary of B0 level. So, population of both levels are comparable at early times. Depending
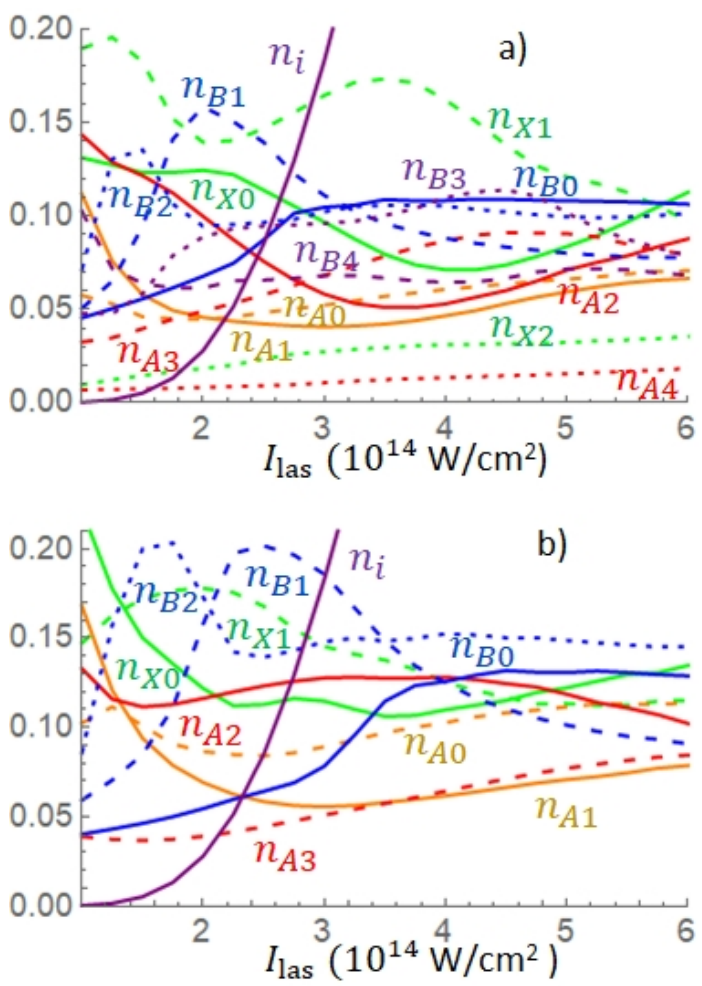

FIG. 3. Dependence of the populations at levels X0 (green, solid), X1 (green, long dashed), X2 (green, dotted), A0 (orange, dashed), A1 (orange, solid), A2 (red, solid), A3 (red, dashed), A4 (red, dotted), B0 (blue, solid), B1 (blue, dashed), B2 (blue, dotted), B3 (purple, dashed), B4 (purple, dotted) normalized to the total ion density, $n_{i}$ on the laser intensity for laser pulse duration of $30 \mathrm{fs}$ and wavelength of $800 \mathrm{~nm}$. Total ionization probability $n_{i} / n_{0}$ is shown with a solid purple line. 13 levels are considered in panel a, four levels have been removed in panel b: X2, A4, B3 and B4.

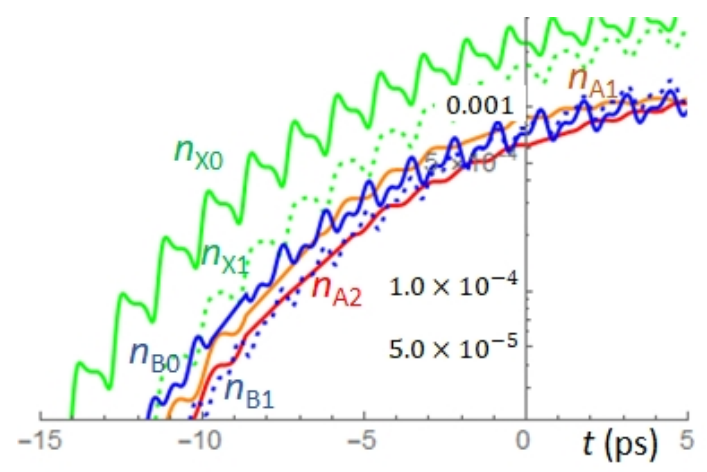

FIG. 4. Time dependence of the populations at states X0 (green, solid), X1 (green, dashed), A1 (orange, solid), A2 (red, solid), B0 (blue, solid) and B1 (blue, dashed) normalized to the total density of neutral molecules. Laser intensity $1.8 \times$ $10^{14} \mathrm{~W} / \mathrm{cm}^{2}$, pulse duration $30 \mathrm{fs}$, wavelength $800 \mathrm{~nm}$ and angle $\Theta=45^{\circ}$. Time $t=0$ corresponds to the maximum of laser intensity. 
on laser parameters, this two-step transfer could be very efficient with population on X1 level being at later times higher than populations at X0 and B levels. Levels A1 and A2 are also populated from X0 level, but their role in populating X1 level is small in the case of linear laser polarization. By contrast, the roles of $\mathrm{B}$ and $\mathrm{A}$ states are exchanged in the case of circular polarization. There, A levels mediate transfer ions to $\mathrm{X} 1$ state and populations at B level are strongly reduced.

\section{B. Linearly polarized laser pulse}

Figure 5 shows distribution of populations over all considered levels in more details for laser pulse duration of 30 fs calculated with the full model including 13 levels. In the ground state $\mathrm{X}$, there is an inversion between levels $\mathrm{X} 0$ and $\mathrm{X} 1$ in all considered interval of laser intensities, while population at level X2 remains rather small, less than 3\%. Populations at levels A0-A3 are of the same order of $5-10 \%$, while population at level A4 is much smaller, less than $2 \%$.

Populations at all levels in B state are of the same order of $10 \%$ with notable variations at low intensities $I_{\text {las }} \lesssim 3 \times 10^{14} \mathrm{~W} / \mathrm{cm}^{2}$ and approximately constant values at higher intensities.

Figure 6 shows populations at five levels of interest in function laser intensity for pulse duration of 10 and 50 fs. No inversion between populations of B0 and X0 states is observed for a short laser pulse (figure 6a). However, population at level X0 gradually decreases with increasing pulse duration, inversion appears already at pulse duration of $30 \mathrm{fs}$ (see figure $3 \mathrm{a}$ and 5) and further increases at pulse duration of 50 fs (figure $6 \mathrm{~b}$ ).

Dependence populations on excited levels on laser wavelength is shown in figure 7 . While B0-X0 inversion is observed for wavelength of $810 \mathrm{~nm}$ (panel a), zone of inversion decreases as wavelength decreases and it is completely suppressed for wavelength of $780 \mathrm{~nm}$ for pulse duration of $30 \mathrm{fs}$. This complicated variation of populations with respect to the laser intensity and wavelength can be explained by a variation of the energy difference between levels under the effect of ponderomotive potential.

This analysis shows that inversion of populations between B0 and X0 levels is unlikely the origin of strong amplification of signals at wavelengths corresponding to B0-X0 and B0-X1 transitions. By contrast, inversion between B0 and A2 and A3 levels is observed in a large range of laser intensities, which favors the hypothesis of amplification in the three level scheme $[12,13]$.

\section{Elliptically polarized laser pulse}

Elliptical polarization of laser pulse removes the degeneracy between transitions to A levels with $m=1$ and $m=-1$. Consequently, transitions to levels $\mathrm{A} v^{+}$and $\mathrm{A} v^{-}$should be considered separately. Figure 8 shows
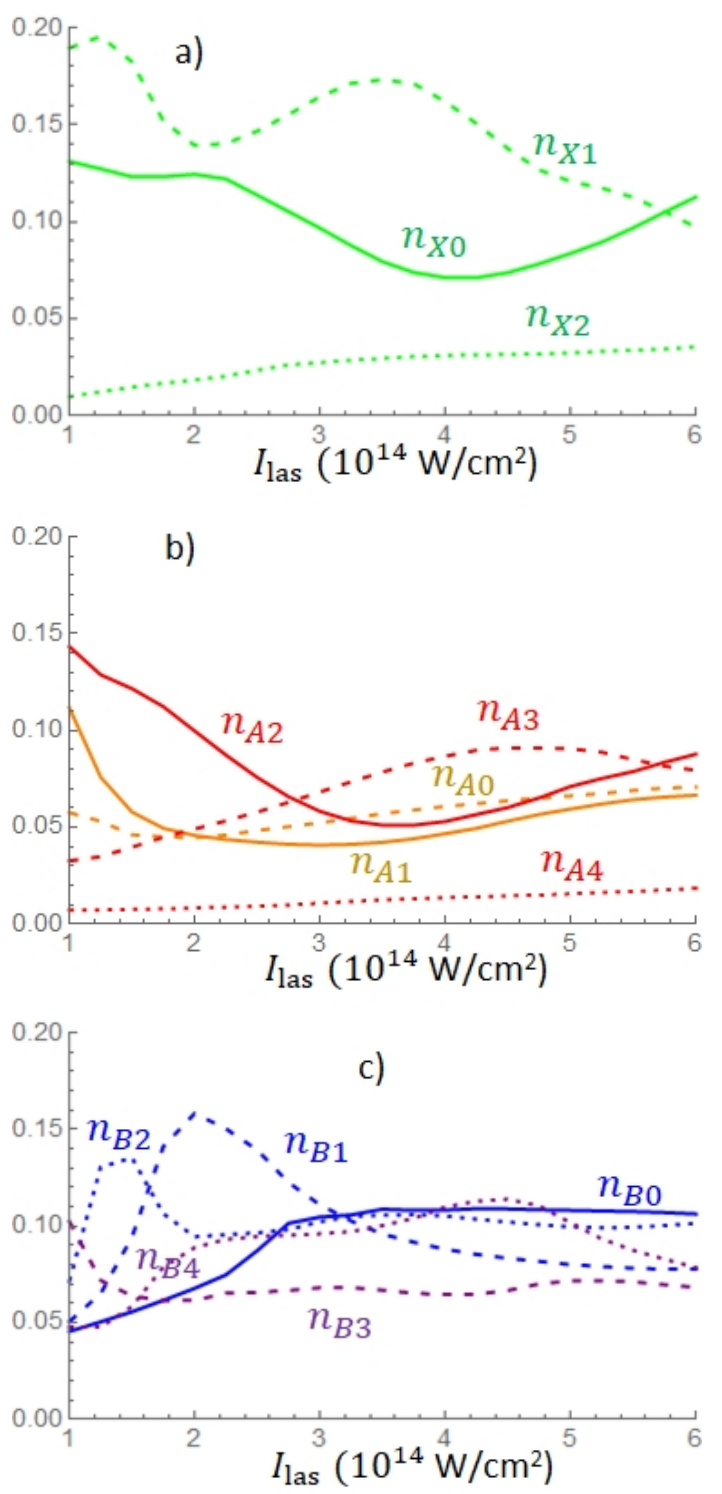

FIG. 5. Dependence of the populations at states X (a), A (b) and B (c), vibration levels are denotes as follows: X0 (green, solid), X1 (green, dashed), X2 (green, dotted), A0 (orange, dashed), A1 (orange, solid), A2 (red, solid), A3 (red, dashed), A4 (red, dotted), B0 (blue, solid), B1 (blue, dashed), B2 (blue, dotted), B4 (purple, dashed) and B4 (purple, dotted) normalized to the total ion density, $n_{i}$ on the laser intensity for laser pulse duration of $30 \mathrm{fs}$ and wavelength of 800 nm.

temporal evolution of populations at A levels for a circularly polarized laser pulse calculated with the model including eighteen levels. Positive ellipticity $\epsilon=1$ favors transition to the states with $m=-1$. A significant level of excitation is achieved already at the laser pulse maximum and evolves further in the second half of the pulse.

Efficiency of ion transition to excited states depends on polarization. As ellipticity increases the rate of tran- 

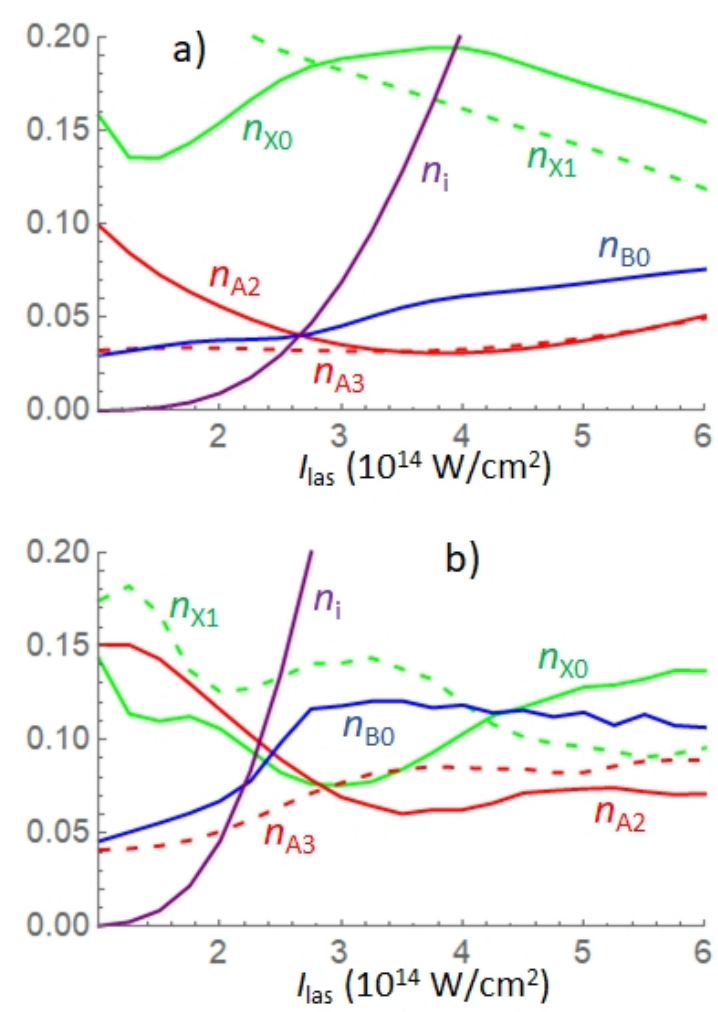

FIG. 6. Dependence of the populations at levels X0 (green, solid), X1 (green, long dashed), A2 (red, solid), A3 (red, dashed) and B0 (blue, solid) normalized to the total ion density, $n_{i}$ on the laser intensity for laser pulse duration $10 \mathrm{fs}$ (a) and $50 \mathrm{fs}$ (b). Total ionization probability $n_{i} / n_{0}$ is shown with a solid purple line. Laser wavelength is $800 \mathrm{~nm}$.

sitions to A states increases while the populations at B states decrease. Figure 9 shows intensity dependence of populations at excited states for linear, elliptical $\epsilon=0.4$ and circular polarization. The case of linear polarization is the same as in figure 3a but only five levels are shown here.

Thus, our analysis shows that circular laser polarization is less suitable for excitation B states. It is more efficiently couples $\mathrm{X}$ and $\mathrm{A}$ states that correspond to magnetic quantum number $m= \pm 1$. This conclusion is supported by studies of dependence of populations at excited states on laser pulse duration (in the range $10-50 \mathrm{fs}$ ) and wavelength (in the range $750-850 \mathrm{~nm}$ ) for circular polarization. The populations at the $\mathrm{B}$ levels are limited to $5-7 \%$, while the populations at $\mathrm{X}$ and $\mathrm{A}$ states vary in the range $5-20 \%$. In some cases an inversion between $\mathrm{A}$ and $\mathrm{B}$ states is observed.

\section{CONCLUSIONS}

We conducted an extensive analysis of ionization and excitation of nitrogen molecules with a high amplitude short laser pulse. The values of populations depend
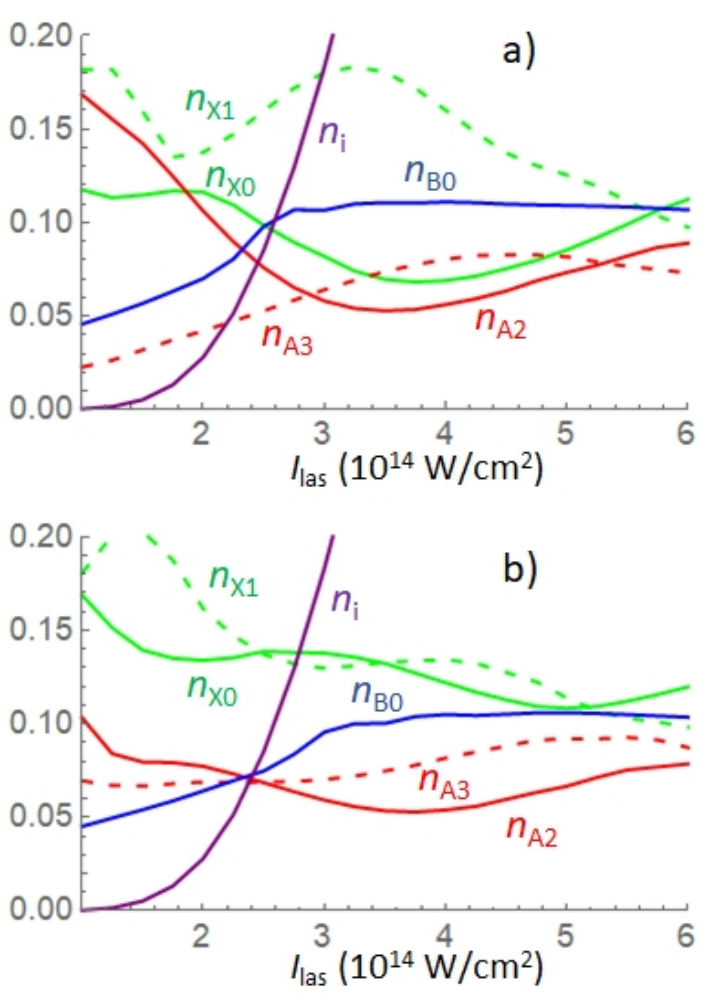

FIG. 7. Dependence of the populations at levels X0 (green,, solid), X1 (green, long dashed), A2 (red, solid), A3 (red, dashed) and B0 (blue, solid) normalized to the total ion density, $n_{i}$ on the laser intensity for laser wavelength of $810 \mathrm{~nm}$ (a) and $780 \mathrm{~nm}$ (b). Total ionization probability $n_{i} / n_{0}$ is shown with a solid purple line. Laser pulse duration is $30 \mathrm{fs}$.

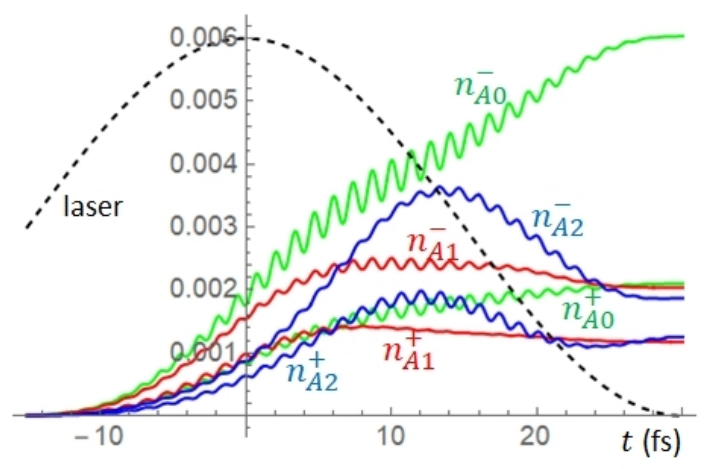

FIG. 8. Dependence of the populations at levels $\mathrm{A} 0^{ \pm}$(green, solid), $\mathrm{A}^{ \pm}$(red, solid) and $\mathrm{A}^{ \pm}$(blue, solid) on time for laser intensity $3 \times 10^{14} \mathrm{~W} / \mathrm{cm}^{2}$, wavelength of $800 \mathrm{~nm}$ and pulse duration of $30 \mathrm{fs}$. Angles of molecule orientation are $\theta_{n}=45^{\circ}$ and $\psi_{n}=90^{\circ}$. Dashed black line shows the temporal evolution of the laser intensity.

strongly on laser parameters and also on the number of considered excited levels. A sufficiently complete description is achieved by considering 11-13 levels covering the range of wavelengths from 300 to $1200 \mathrm{~nm}$. This includes $2-3$ vibrational levels in the ground state, $4-5$ levels in the 

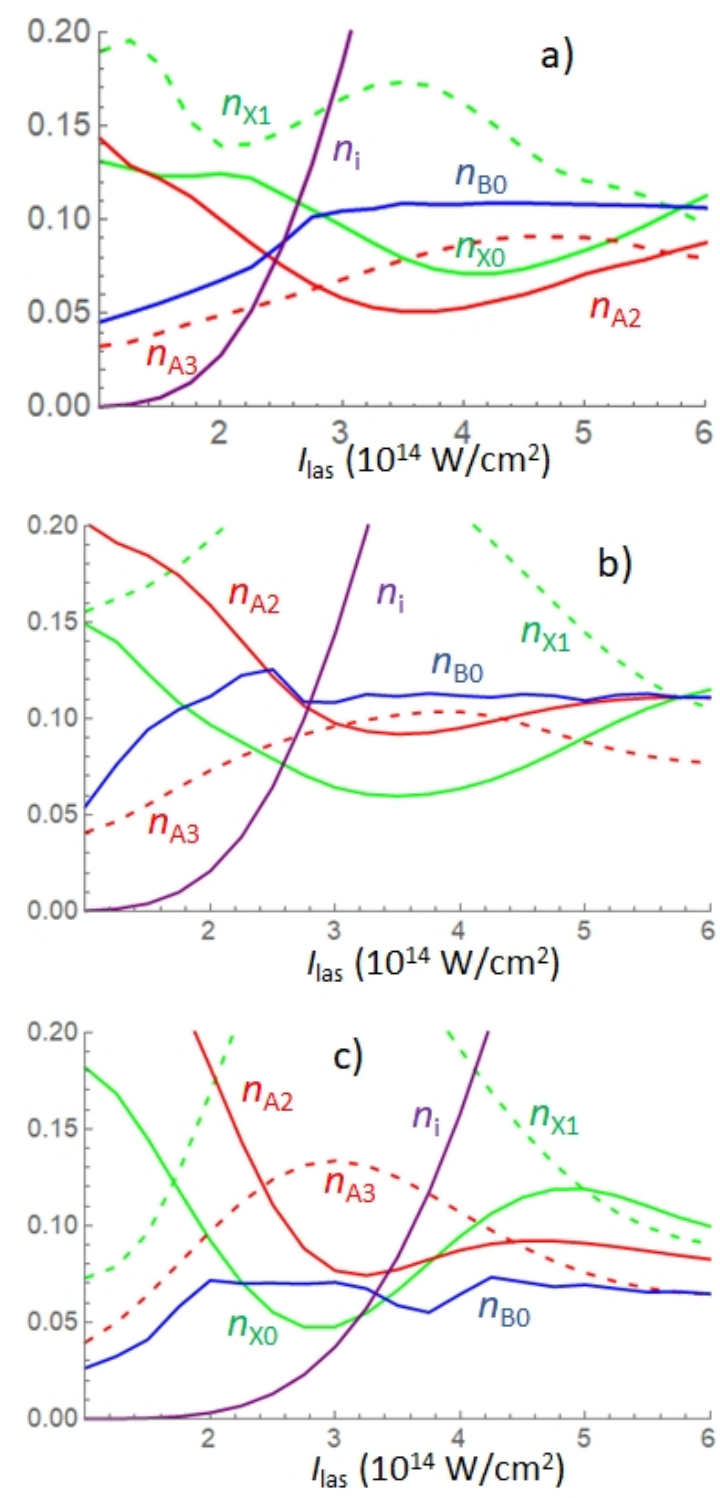

FIG. 9. Dependence of the populations at levels X0 (green, solid), X1 (green, dashed), A2 (red, solid), A3 (red, dashed) and B0 (blue, solid) on laser intensity for wavelength of $800 \mathrm{~nm}$ and pulse duration of $30 \mathrm{fs}$. Panel a: linear polarizaion, b: $\epsilon=0.4$, c: circular polarization. Curves A2 and A3 report cumulative populations at the right and left-handed states.

A state and 4-5 levels in the B state.

A theoretical approach based on the density matrix formalism has an advantage compared to the equations for the probability amplitudes by accounting at the same time for the ionization and excitation processes. It is therefore, quantitatively more accurate and capable for predicting population dependence on laser intensity, wavelength and pulse duration.
Ionization model proposed by Tong et al. [36] agrees well with the experimental data. The ionization probability is reduced by a constant factor of 10 due to averaging over radial intensity profile of laser pulse. Ionization to the ground level X0 dominates. Excitation to higher electronic and vibrational levels is due to a dipole coupling of excited levels to the ground state in a strong laser electric field. For linearly polarized laser pulse, transitions to B state dominate. This is explained by the preference of transitions with the same magnetic quantum number $m=0$. By contrast, a circularly polarized laser pulse favors transition with $\Delta m= \pm 1$, which leads to increase of populations in A states and decrease in B states.

We found, however, a significant quantitative difference in the populations presented in reference [26], where the authors used very similar approach, except the angle averaging. The origins of this difference need further investigation. Averaging the results over molecule orientation with respect to the laser field orientation is an important part of the model. By choosing appropriate orientation one may enable or disable B-X and A-X transitions and, therefore, incorrectly evaluate to the population partition. We performed averaging by assuming an isotropic molecule distribution. It would be interesting to control the populations by using a aligned nitrogen gas in experiments.

Ionization-excitation model predicts a robust inversion between B and A states in a broad range of laser intensities and pulse duration considered. By contrast, population inversion between $\mathrm{B} 0$ and $\mathrm{X} 0$ and $\mathrm{X}$ levels with vibrational numbers $v \geq 1$ is found to exist only in a very limited range of parameters. This fact indicates that amplification without inversion in the $\mathrm{V}$ scheme is the most plausible explanation of observed B0-X0 and B0-X1 emissions at 391 and $428 \mathrm{~nm}$ [13].

\section{ACKNOWLEDGMENTS}

The work is supported in part by the National Natural Science Foundation of China (Grants No. 12034013), Innovation Program of Shanghai Municipal Education Commission (Grant No. 2017-01-07-00-07-E00007).

\section{Appendix A: Parameters of the nitrogen molecular ion}

Dipole moments are expressed in the atomic units, $e a_{\mathrm{B}}$. According to equation (12), expressions for dipole moment through the Einstein coefficient read:

$$
\mu_{a b}=0.99 \times 10^{-3}\left(A_{a b} \lambda_{a b}^{3}\right)^{1 / 2},
$$

where $A_{a b}$ is in s ${ }^{-1}$ and the wavelength $\lambda_{a b}$ in microns. 


\begin{tabular}{|c|c|c|c|c|}
\hline Transition & $\lambda_{a b}, \mathrm{~nm}$ & $A_{a b}, \mathrm{~s}^{-1}$ & $\omega_{a b} / \omega_{a t}$ & $\mu_{a b} / e a_{\mathrm{B}}$ \\
\hline $\mathrm{A}(0)-\mathrm{X}(0)$ & 1108.7 & $4.63 \times 10^{4}$ & 0.0415 & 0.249 \\
\hline $\mathrm{A}(0)-\mathrm{X}(1)$ & 1461.0 & $1.46 \times 10^{4}$ & 0.0315 & 0.211 \\
\hline $\mathrm{A}(0)-\mathrm{X}(2)$ & 2126.5 & $1.37 \times 10^{3}$ & 0.0216 & 0.114 \\
\hline $\mathrm{A}(1)-\mathrm{X}(0)$ & 918.15 & $5.70 \times 10^{4}$ & 0.0501 & 0.208 \\
\hline $\mathrm{A}(1)-\mathrm{X}(1)$ & 1147.2 & $3.17 \times 10^{3}$ & 0.0401 & 0.068 \\
\hline $\mathrm{A}(1)-\mathrm{X}(2)$ & 1521.1 & $1.17 \times 10^{4}$ & 0.0302 & 0.201 \\
\hline $\mathrm{A}(2)-\mathrm{X}(0)$ & 785.25 & $3.86 \times 10^{4}$ & 0.0585 & 0.135 \\
\hline $\mathrm{A}(2)-\mathrm{X}(1)$ & 946.69 & $3.68 \times 10^{4}$ & 0.0486 & 0.175 \\
\hline $\mathrm{A}(2)-\mathrm{X}(2)$ & 1188.0 & $1.32 \times 10^{3}$ & 0.0387 & 0.047 \\
\hline $\mathrm{A}(3)-\mathrm{X}(0)$ & 687.36 & $1.94 \times 10^{4}$ & 0.0669 & 0.079 \\
\hline $\mathrm{A}(3)-\mathrm{X}(1)$ & 808.18 & $5.27 \times 10^{4}$ & 0.0569 & 0.165 \\
\hline $\mathrm{A}(3)-\mathrm{X}(2)$ & 977.40 & $1.25 \times 10^{4}$ & 0.0470 & 0.107 \\
\hline $\mathrm{A}(4)-\mathrm{X}(0)$ & 612.30 & $8.17 \times 10^{3}$ & 0.0751 & 0.043 \\
\hline $\mathrm{A}(4)-\mathrm{X}(1)$ & 706.37 & $4.13 \times 10^{4}$ & 0.0651 & 0.119 \\
\hline $\mathrm{A}(4)-\mathrm{X}(2)$ & 832.31 & $4.30 \times 10^{4}$ & 0.0552 & 0.156 \\
\hline $\mathrm{B}(0)-\mathrm{X}(0)$ & 391.44 & $1.24 \times 10^{7}$ & 0.1175 & 0.854 \\
\hline $\mathrm{B}(0)-\mathrm{X}(1)$ & 427.81 & $3.70 \times 10^{6}$ & 0.1075 & 0.533 \\
\hline $\mathrm{B}(0)-\mathrm{X}(2)$ & 470.92 & $7.34 \times 10^{5}$ & 0.0976 & 0.274 \\
\hline $\mathrm{B}(1)-\mathrm{X}(0)$ & 358.21 & $5.86 \times 10^{6}$ & 0.1283 & 0.514 \\
\hline $\mathrm{B}(1)-\mathrm{X}(1)$ & 388.43 & $4.87 \times 10^{6}$ & 0.1084 & 0.529 \\
\hline $\mathrm{B}(1)-\mathrm{X}(2)$ & 423.65 & $4.40 \times 10^{6}$ & 0.1085 & 0.572 \\
\hline $\mathrm{B}(2)-\mathrm{X}(0)$ & 330.80 & $7.90 \times 10^{5}$ & 0.1390 & 0.167 \\
\hline $\mathrm{B}(2)-\mathrm{X}(1)$ & 356.39 & $8.25 \times 10^{5}$ & 0.1290 & 0.605 \\
\hline $\mathrm{B}(2)-\mathrm{X}(2)$ & 385.79 & $1.50 \times 10^{6}$ & 0.1192 & 0.291 \\
\hline $\mathrm{B}(3)-\mathrm{X}(0)$ & 307.82 & $2.02 \times 10^{4}$ & 0.1494 & 0.024 \\
\hline $\mathrm{B}(3)-\mathrm{X}(1)$ & 329.87 & $1.77 \times 10^{6}$ & 0.1394 & 0.250 \\
\hline $\mathrm{B}(3)-\mathrm{X}(2)$ & 354.89 & $8.80 \times 10^{6}$ & 0.1295 & 0.621 \\
\hline $\mathrm{B}(4)-\mathrm{X}(1)$ & 307.64 & $6.23 \times 10^{4}$ & 0.1494 & 0.042 \\
\hline $\mathrm{B}(4)-\mathrm{X}(2)$ & 329.34 & $2.35 \times 10^{6}$ & 0.1396 & 0.287 \\
\hline & & & & \\
\hline
\end{tabular}

TABLE II. Wavelengths $\lambda_{a b}$, the corresponding Einstein coefficients $A_{a b}$, frequencies and dipole moments for the transitions $\mathrm{AX}$ and $\mathrm{BX}$ of the molecular ion $\mathrm{N}_{2}^{+}$. Here, $\omega_{a t}=v_{a t} / a_{\mathrm{B}}$ is the atomic frequency and $v_{a t}=e^{2} / 4 \pi \epsilon_{0} \hbar$ is the atomic velocity; $\mu_{a b}$ is calculated from equation (A1) by using the Einstein coefficients given in references [24, 25, 27, 28].

\begin{tabular}{|c|c|c|c||c|c|}
\hline $\mathrm{N}_{2}^{+}$orbital & $l$ & $m$ & $C_{l}$ & $v$ & $q_{a}^{\mathrm{FCi}}$ \\
\hline \multirow{3}{*}{$\mathrm{X}^{2} \Sigma_{g}^{+}$} & 0 & 0 & 2.86 & 0 & 0.905 \\
\cline { 2 - 6 } & 2 & 0 & 1.37 & 1 & 0.089 \\
\cline { 2 - 6 } & 4 & 0 & 0.21 & 2 & 0.006 \\
\hline \multirow{3}{*}{$\mathrm{A}^{2} \Pi_{u}$} & 1 & \pm 1 & 3.06 & 0 & 0.275 \\
\cline { 2 - 6 } & 3 & \pm 1 & 0.21 & 1 & 0.320 \\
\cline { 2 - 6 } & & & & 2 & 0.215 \\
\cline { 2 - 6 } & & & & 3 & 0.111 \\
\hline \multirow{3}{*}{$\mathrm{B}^{2} \Sigma_{u}^{+}$} & 1 & 0 & 4.23 & 0 & 0.049 \\
\cline { 2 - 6 } & & 0 & 0.83 & 1 & 0.886 \\
\cline { 2 - 6 } & & & & 2 & 0.111 \\
\hline
\end{tabular}

TABLE III. Coefficients for $\mathrm{N}_{2}$ molecule ionization in the ground and excited states [26] and Franck-Condon ionization factors [28].

[1] Y. Yao, G. Li, C. Jing, B. Zeng, W. Chu, J. Ni, H. Zhang, H. Xie, C. Zhang, H. Li, H. Xu, S. L. Chin, Y. Cheng, and $\mathrm{Z}$. $\mathrm{Xu}$. Remote creation of coherent emissions in air with two-color ultrafast laser pulses. New J. Phys., 15: 023046, 2013. 
[2] Y. Liu, Y. Brelet, G. Point, A. Houard, and A. Mysyrowicz. Self-seeded lasing action of air pumped by $800 \mathrm{~nm}$ femtosecond laser pulses. Opt. Express, 21:22791, 2013.

[3] G. Li, C. Jing, B. Zeng, H. Xie, J. Yao, W. Chu, J. Ni, H. Zhang, H. Xu, Y. Cheng, and Z. Xu. Signature of superradiance from a nitrogen-gas plasma channel produced by strong-field ionization. Phys. Rev. A, 89:033833, 2014.

[4] G. Point, Y. Liu, Y. Brelet, S. Mitryukovskiy, P. J. Ding, A. Houard, and A. Mysyrowicz. Lasing of ambient air with microjoule pulse energy pumped by a multi-terawatt femtosecond laser. Opt. Lett., 39:1725, 2014.

[5] H. Xu, E. Lötstedt, A. Iwasaki, and K. Yamanouchi. Sub10-fs population inversion in $\mathrm{N}_{2}^{+}$in air lasing through multiple state coupling. Phys. Rev. Lett., 125:053201, 2020.

[6] J. Yao, S. Jiang, W. Chu, B. Zeng, C. Wu, R. Lu, Z. Li, H. Xie, G. Li, C. Yu, Z. Wang, H. Jiang, Q. Gong, and Y. Cheng. Population redistribution among multiple electronic states of molecular nitrogen ions in strong laser fields. Phys. Rev. Lett., 116:143007, 2016.

[7] Y. Zhang, E. Lötstedt, and K. Yamanouchi. Population inversion in a strongly driven two-level system far-off resonance. J. Phys. B: At. Mol. Phys., 50:185603, 2017.

[8] M. Britton, P. Laferrière, D. H. Ko, Z. Li, F. Kong, G. Brown, A. Naumov, C. Zhang, L. Arissian, and P. B. Corkum. Testing the role of recollision in $\mathrm{N}_{2}^{+}$air lasing. Phys. Rev. Lett., 120:133208, 2018.

[9] M. Britton, M. Lytova, P. Laferrière, P. Peng, F. Morales, D. H. Ko, M. Richter, P. Polynkin, D. M. Villeneuve, C. Zhang, M. Ivanov, M. Spanner, L. Arissian, and P. B. Corkum. Short- and long-term gain dynamics in $\mathrm{N}_{2}^{+}$air lasing. Phys. Rev. A, 100:013406, 2019.

[10] Y Liu, P Ding, G Lambert, A Houard V T Tikhonchuk, and A Mysyrowicz. Recollision-induced superradiance of ionized nitrogen molecules. Phys. Rev. Lett., 115:133203, 2015 .

[11] V. T. Tikhonchuk, J.-F. Tremblay-Bugeaud, Y. Liu, A. Houard, and A. Mysyrowicz. Excitation of nitrogen molecular ions in a strong laser field by electron recollisions. Europ. J. Phys. D, 71:292, 2017.

[12] V. T. Tikhonchuk, Y. Liu, R. Danylo, A. Houard, and A. Mysyrowicz. Theory of femtosecond strong field ion excitation and subsequent lasing in $\mathrm{N}_{2}^{+}$. New J. Phys., 23:023035, 2021.

[13] A. Mysyrowicz, R. Danylo, A. Houard, V. Tikhonchuk, X. Zhang, Z. Fan, Q. Liang, S. Zhuang, L. Yuan, and Y. Liu. Lasing without population inversion in $\mathrm{N}_{2}^{+}$. APL Photonics, 4:110807, 2019.

[14] O. A. Kocharovskaya and Ya. I. Khanin. Coherent amplification of an ultrashort pulse in a three-level medium without a population inversion. J. Exp. Theor. Phys. Lett., 48:630, 1988.

[15] S. E. Harris. Lasers without inversion; interference of lifetime-broadened resonances. Phys. Rev. Lett., 62:1033, 1988.

[16] M. O. Scully, S.-Y. Zhu, and A. Gavrielides. Degenerate quantum-beat laser: lasing without inversion and inversion without lasing. Phys. Rev. Lett., 62:2813, 1989.

[17] A. A. Svidzinsky, L. Yuan, and M. O. Scully. Transient lasing without inversion. New J. Phys., 15:053044, 2013.

[18] J. Mompart, C. Peters, and R. Corbalan. Interpretation of transient $\mathrm{V}$ scheme amplification without inversion. Quantum Semiclass. Opt., 10:355, 1998.
[19] V. A. Malyshev, I. V. Ryzhov, E. D. Trifonov, and A. I. Zaitsev. Superradiance without inversion. Laser Phys., 8:494, 1998.

[20] V. V. Kozlov, P. G. Polynkin, and M. O. Scully. Resonant raman amplification of ultrashort pulses in a $\mathrm{V}$-type medium. Phys. Rev. A, 59:3060, 1999.

[21] H. Zhang, C. Jing, J. Yao, G. Li, B. Zeng, W. Chu, J. Ni, H. Xie, H. Xu, S. L. Chin, K. Yamanouchi, Y. Cheng, and $\mathrm{Z}$. $\mathrm{Xu}$. Rotational coherence encoded in an "airlaser" spectrum of nitrogen molecular ions in an intense laser field. Phys. Rev. X, 3:041009, 2013. doi: 10.1103/PhysRevX.3.041009.

[22] M. Richter, M. Lytova, F. Morales, S. Haessler, O. Smirnova, M. Spanner, and M. Ivanov. Rotational quantum beat lasing without inversion. Optica, 7:586, 2020. doi:10.1364/OPTICA.390665.

[23] M. Lytova, M. Richter, F. Morales, O. Smirnova, M. Ivanov, and M. Spanner. $\mathrm{N}_{2}^{+}$lasing: Gain and absorption in the presence of rotational coherence. Phys. Rev. A, 2020.

[24] S. R. Langhoff, Jr. C. W. Bauschlicher, and H. Partridge. Theoretical study of the $\mathrm{N}_{2}^{+}$Meinel system. J. Chem. Phys., 87:4716, 1987.

[25] S. R. Langhoff and Jr. C. W. Bauschlicher. Theoretical study of the first and second negative systems of $\mathrm{N}_{2}^{+} . J$. Chem. Phys., 88:329, 1988.

[26] Q. Zhang, H. Xie, G. Li, X. Wang, H. Lei, J. Zhao, Z. Chen, J. Yao, Y. Cheng, and Z. Zhao. Sub-cycle coherent control of ionic dynamics via transient ionization injection. Comm. Phys., 3:50, 2020. doi:10.1038/s42005020-0321-7.

[27] D. C. Jain and R. C. Sahni. Einstein coefficients, cross sections, $f$ values, dipole moments, and all that. Int. J. Quantum Chem., 1:721, 1967.

[28] A. Lofthus and P. H. Krupenie. The spectrum of molecular nitrogen. J. Phys. Chern. Ref. Data, 6:113, 1977.

[29] Y. Fu, E. Lötstedt, H. Li, S. Wang, D. Yao, T. Ando, A. Iwasaki, F. H. M. Faisal, K. Yamanouchi, and H. Xu. Optimization of $\mathrm{N}_{2}^{+}$lasing through population depletion in the $\mathrm{X}_{2} \sigma_{g}^{+}$state using elliptically modulated ultrashort intense laser fields. Phys. Rev. Res., 2:012007(R), 2020.

[30] H. Li, E. Lötstedt, H. Li, Y. Zhou, N. Dong, L. Deng, P. Lu, T. Ando, A. Iwasaki, Y. Fu, S. Wang, J. Wu, K. Yamanouchi, and $\mathrm{H}$. Xu. Giant enhancement of air lasing by complete population inversion in $\mathrm{N}_{2}^{+}$. Phys . Rev. Lett., 125:053201, 2020.

[31] J. Chen, J. Yao, H. Zhang, Z. Liu, B. Xu, W. Chu, L. Qiao, Z. Wang, J. Fatome, O. Faucher, C. Wu, and Y. Cheng. Electronic-coherence-mediated molecular nitrogen-ion lasing in a strong laser field. Phys. Rev. A, 100:031402(R), 2019.

[32] R.C. Hilborn. Einstein coefficients, cross sections, $f$ values, dipole moments, and all that. Am. J. Phys., 50:982, 1982.

[33] A. M. Perelomov, V. S. Popov, and M. V. Terent'ev. Ionization of atoms in an alternating electric field. Sov. Phys. JETP, 23:924, 1966.

[34] M. V. Ammosov, N. B. Delone, and V. P. Krainov. Tunnel ionization of complex atoms and atomic ions in electromagnetic field. Sov. Phys. JETP, 64:1191, 1986.

[35] V. S. Popov. Tunnel and multiphoton ionization of atoms and ions in a strong laser field (Keldysh theory). Physics - Uspekhi, 47:855, 2004. 
[36] X. M. Tong, Z. X. Zhao, and C. D. Lin. Theory of molecular tunneling ionization. Phys. Rev. A, 66:033402, 2002.

[37] L. D. Landau and E. M. Lifshitz. Quantum Mechanics (Non-relativistic theory) (Corse of theoretical physics; v.3). Butterworth-Heinemann, Elsevier Ltd., Oxford, 1977. ISBN 0750635398.
[38] C. Guo, M. Li, J. P. Nibarger, and G. N. Gibson. Single and double ionization of diatomic molecules in strong laser fields. Phys. Rev. A, 58:R4271, 1998.

[39] S. Petretti, Y. V. Vanne, A. Saenz, A. Castro, and P. Decleva. Alignment-dependent ionization of $\mathrm{N}_{2}, \mathrm{O}_{2}$, and $\mathrm{CO}_{2}$ in intense laser fields. Phys. Rev. Lett., 104:223001, 2010. 